\title{
Persistent Edge Current in the Fractional Quantum Hall Effect
}

\author{
Kazusumi Ino * \\ Institute for Solid State Physics, University of Tokyo, \\ Roppongi 7-22-1, Minatoku, Tokyo, 106, Japan
}

\begin{abstract}
We study the persistent edge current in the fractional quantum Hall effect. We give the grand partition functions for edge excitations of hierarchical states coupled to an Aharanov-Bohm flux and derive the exact formula of the persistent edge current. For $m$-th hierarchical states with $m>1$, it exhibits anomalous oscillations in its flux dependence at low temperatures. The current as a function of flux goes to the sawtooth function with period $\phi_{0} / m$ in the zero temperature limit. This phenomenon provides a new evidence for exotic condensation in the fractional quantum Hall effect. We propose experiments of measuring the persistent edge current to confirm the existence of the hierarchy.

PACS: $73.40 \mathrm{Hm}, 74.20-\mathrm{z}$.
\end{abstract}

The predictions of the edge theory [1],2] of the Fractional Quantum Hall (FQH) effect have been confirmed by tunneling experiments [3] for $\nu=1 / q$ ( $q$ : odd integer). However a recent tunneling experiment 位 for more general filling fractions shows no evidence for the existence of the hierarchy [5.6]. It is desirable to have other methods other than tunneling for the confirmation of the hierarchy. Among others, the Aharanov-Bohm effect on the edge state may be a good candidate.

Recently the Aharanov-Bohm effect (AB effect) on the edge state of the $\mathrm{FQH}$ effect attracted attentions [7,8]. Especially, the amplitude of the persistent edge current induced by the $\mathrm{AB}$ flux was calculated for the $\nu=\frac{1}{q}$ Laughlin state by Geller et al [8]. It was shown that the current is periodic with period $\phi_{0}=h c / e$ as required by the general theorem of Byers-Young [9]. This result is consistent with the microscopic study by Thouless and Gefen [10]. As they argued, the existence of the fractionally charged quasiparticle is necessary for that period.

In [11], we investigated the effect of pairing on the persistent edge current of quantum Hall analogs of BCS superconductor, so called paired FQH states [12 14] in which the electrons with an even number of flux (composite fermions) are paired. We found anomalous oscillations of the current at low temperature. They converge to the sawtooth function with period $\phi_{0} / 2$ at zero temperature, which is an indication of a pair condensation. This phenomenon was explained as follows: although the the bulk of paired states are BCS condensate of composite fermions, the condensation in the edge never occurs at finite temperature from the Mermin-Wagner theorem (no spontaneous symmetry breaking in $1+1$ dimensions) 17 . Thus the phenomenon provides an interesting bridge between the BCS condensation in $2+1$ dimensions and the one in $1+1$ dimension.

On the other hand, it is known that composite bosonic objects of electron and an odd number of flux (composite bosons) are condensated in the Laughlin states and the hierarchical states [18,19. It is naturally suggested that similar phenomenon may occur in the hierarchical states. In this paper, we study the persistent edge current in the Jain' hierarchy [5], which is by now a standard picture for the FQH effect. We address some other issues along with it.

Let us consider a single edge of a FQH state coupled to an $\mathrm{AB}$ flux. For example, it is possible to change the strength of magnetic field for a FQH droplet within a given plateau. In the composite boson picture, the situation is similar to flux piercing in a type II superconductor. Also we can consider the boundary of an antidot in the Hall bar.

We first clarify the excitation structure on a single edge state in the general hierarchical scheme [20,15]. Edge excitations of a state of $m$-th hierarchy involve $m$ species of chiral bosons $\varphi_{i}(i=1, \cdots, m)$. Their couplings are specified by an integer matrix $K$ 20.15. It is actually a metric of a lattice $\Gamma$, which is the lattice of the electron field

$$
e^{i \mathbf{v} \cdot \phi}, \quad \mathbf{v}=\sum_{a=1}^{m} v^{a} \mathbf{e}^{a}, \quad v^{a} \in \mathbf{Z} .
$$

Here $\mathbf{e}_{a}(a=1, \cdots, m)$ form a basis of the lattice $\Gamma$ with $\mathbf{e}_{a} \mathbf{e}_{b}=K_{a b}$. The filling fraction of the state is given by

$$
\nu=\sum_{a, b=1}^{m}\left(K^{-1}\right)_{a b} .
$$

From the no monodromy condition between edge excitations and electrons, edge excitations are labeled by the dual lattice $\Gamma^{*}$ of $\Gamma$. $\Gamma^{*}$ is spanned by the dual basis $\mathbf{e}_{a}^{*}$ with $\mathbf{e}_{a}^{*} \mathbf{e}_{b}=\delta_{a b}$. Thus $\mathbf{w} \in \Gamma^{*}$ is a vector which satisfies

*e-mail:ino@kodama.issp.u-tokyo.ac.jp 
$\mathbf{w} \cdot \mathbf{v} \in \mathbf{Z}$. The energy (conformal weight) $J$ and charge $Q$ of quasiparticle $e^{i \mathbf{w} \varphi}$ are given by

$$
J=\frac{1}{2} \mathbf{w} \cdot \mathbf{w}, \quad Q=\mathbf{t} \cdot \mathbf{w} .
$$

Here $\mathbf{t}$ is a real vector of charge unit with $t_{a}=1 / K_{a a}$. From these formulas, Fermi statistics of electrons requires $K_{a a}$ to be odd. By moding out the electrons, we see that fractionally charged quasiparticles are characterized a lattice $\Gamma^{*} / \Gamma$. The lattice $\Gamma^{*} / \Gamma$ has $\operatorname{det} K$ elements, which determines the topological order.

The Jain's hierarchical scheme is obtained by taking $K$ as

$$
K_{a b}=\delta_{a b}+s C_{a b},
$$

where $s$ is a positive even integer and $C_{a b}=1$ for $\forall a, b=1, \cdots, m$. In this case, as $\operatorname{det} K=m s+1$, there are thus $m s+1$ sectors. From (3), the filling fraction is given by

$$
\nu=\frac{m}{m s+1} .
$$

The complete description of the edge excitations can be given by a Virasoro character which corresponds to the partition function of the grand ensemble of quasiparticles. Summing up the characters for the points in $\Gamma^{*}$, we get the grand partition function as

$$
Z(\tau)=\frac{1}{\eta^{m}} \sum_{\mathbf{w} \in \Gamma^{*}} \exp \left[2 \pi i \tau\left(\frac{1}{2} \mathbf{w} \cdot \mathbf{w}\right)\right],
$$

where $\eta$ is the Dedekind function $\eta(\tau)=x^{\frac{1}{24}} \prod_{n=1}^{\infty}(1-$ $\left.x^{n}\right), \quad x=e^{2 \pi i \tau}$ and $\tau=i \frac{T_{0}}{T} . \quad T_{0}=\frac{\hbar v}{k_{B} L}$ is a temperature scale induced by $L$ the circumference of the edge state and $v$ the Fermi velocity of the edge modes. For example, a Fermi velocity of $10^{6} \mathrm{~cm} / \mathrm{s}$ and circumference of $1 \mu \mathrm{m}$ yields $T_{0} \sim 60 \mathrm{mK}$.

Let us consider the effect of additional AB flux $\Phi$ for the hierarchical state. For that end, we note that the $\mathrm{AB}$ flux only couples to the charge degrees of freedom. Thus we need to decompose $\Gamma^{*}$ into the neutral degrees of freedom and the charge degrees of freedom explicitly.

In $\Gamma^{*}$, the sublattice $\Gamma_{0}^{*}$ formed by points $\mathbf{t} \cdot \mathbf{w}=0$ is a $m-1$ dimensional lattice, which account for the neutral degrees of freedom. By a suitable $m-1$ dimensional modular transformation, the matrix $K_{a b}$ on $\Gamma_{0}^{*}$ is equivalent to the Cartan matrix of $\mathrm{SU}(m)$ 20]. Actually, from (3), we see that there are $m(m-1)$ neutral excitations with $J=1$. As shown by Fröhlich and Zee [15], these excitations form the affine $\mathrm{SU}(m)$ algebra of level 1 and enlarge the chiral algebra. The states in $\Gamma^{*}$ are decomposed into $m$ integrable representations of $\mathrm{SU}(m)_{1}$, which can be labeled by $a=1, \cdots, m$. They correspond to $a$-th antisymmetric tensor of $\mathrm{SU}(m)$ respectively. Note that the electrons carry the quantum number of the fundamental representation of $\mathrm{SU}(m)$, which corresponds to Jain's idea of dividing the electron into $m$ species [5].
To decompose $\Gamma^{*}$ into the neutral $\mathrm{SU}(m)_{1}$ degrees of freedom and the charge degrees of freedom, we consider excitations which form a fundamental representation of $\mathrm{SU}(m)$ with the charge $\frac{1}{m} \nu$. They are 'quark-like' excitations, generating all the excitations. The quantum number for a composite state of them is obtained by the fusion rules of $\mathrm{SU}(m)_{1}$. The fusion rules of $\mathrm{SU}(m)_{1}$ is given by

$$
a_{1} \times a_{2}=a_{1}+a_{2} \quad(\bmod m),
$$

which has a structure of $\mathbf{Z} / m \mathbf{Z}$. By using (7), it is readily seen that a state which is in $a$ under $\mathrm{SU}(m)_{1}$ has a charge $\frac{a}{m} \nu$ up to $\mathbf{Z} \nu$.

Under these quantum numbers of $\mathrm{SU}(m)$ and $\mathrm{U}(1)$, we can reorganize the sum in (6) as

$$
Z(\tau)=\sum_{a=1}^{m} \chi_{a / m}(\nu \tau) \chi_{a}^{\mathrm{SU}(m)_{1}}(\tau),
$$

where $\chi_{a}^{\mathrm{SU}(m)_{1}}(\tau)$ is the character for representation $a$ of affine $\mathrm{SU}(m)_{1}$ :

$$
\begin{aligned}
\chi_{a}^{\mathrm{SU}(m)_{1}}(\tau)=\mathrm{e}^{-\pi i \tau \frac{a^{2}}{m}} \times \\
\quad \times \frac{1}{\eta^{m-1}} \sum_{n_{1}+\cdots+n_{m}=a} \mathrm{e}^{\pi i \tau\left(n_{1}^{2}+n_{2}^{2}+\cdots+n_{m}^{2}\right)} .
\end{aligned}
$$

Also $\chi_{a / m}(\tau)$ is defined as

$$
\chi_{a / m}(\tau)=\frac{1}{\eta} \sum_{n \in \mathbf{Z}} \mathrm{e}^{\pi \tau i(n+a / m)^{2}} .
$$

Similar decomposition of partition function has been given for the annulus case [16].

Having separated the contribution from the charge degrees of freedom in $Z(\tau)$, let us consider the $\mathrm{AB}$ effect. $\mathrm{An} A B$ flux causes a spectral flow in charge spectrum which results in the change in $\chi_{a / m}(\nu \tau)$ as

$$
\begin{aligned}
\chi_{a / m}(\nu \tau, \phi) & =\frac{1}{\eta} \sum_{n \in \mathbf{Z}} \mathrm{e}^{\pi \tau i \nu(n+a / m-\phi)^{2}} \\
& \equiv \frac{1}{\eta} \Theta\left[\begin{array}{c}
0 \\
(m-a) / m
\end{array}\right](\phi \mid t / \nu) .
\end{aligned}
$$

where $\phi=\Phi / \phi_{0}$ with $\phi_{0}=h c / e$ the unit flux quantum and $t=-\frac{1}{\tau}=i \frac{T}{T_{0}} . \Theta\left[\begin{array}{l}a \\ b\end{array}\right]$ is the generalized $\Theta$ function [21]. Thus the grand partition function with the $\mathrm{AB}$ flux becomes

$$
Z(\tau, \phi)=\sum_{a=1}^{m} \frac{1}{\eta} \Theta\left[\begin{array}{c}
0 \\
a / m
\end{array}\right](\phi \mid t / \nu) \chi_{m-a}^{\mathrm{SU}(m)_{1}}(\tau) .
$$

Obviously $Z(\tau, \phi)$ is a periodic function of $\phi$ with period $\phi_{0}$. 
The exact formula for the persistent edge current is readily deduced from (13). In general, the persistent current $I$ is defined by the following formula:

$$
I \equiv \frac{T}{\phi_{0}} \frac{\partial \ln Z(\tau, \phi)}{\partial \phi} .
$$

From (14) and (13) we get the amplitude of persistent current induced by $\phi$ as

$$
I(\tau, \phi)=\frac{T}{\phi_{0}} \frac{\sum_{\alpha=1}^{m} \Theta^{\prime}\left[\begin{array}{c}
0 \\
a / m
\end{array}\right](\phi \mid t / \nu) \chi_{m-a}^{\mathrm{SU}(m)_{1}}(\tau)}{\sum_{\alpha=1}^{m} \Theta\left[\begin{array}{c}
0 \\
a / m
\end{array}\right](\phi \mid t / \nu) \chi_{m-a}^{\mathrm{SU}(m)_{1}}(\tau)}
$$

where $\Theta^{\prime}$ denotes the differentiation of $\Theta$ in $\phi$. For example, the persistent current in the Laughlin state $(m=1)$ is calculated to be

$$
I_{\text {Laugh }}=\frac{2 \pi T}{\phi_{0}} \sum_{n=0}^{\infty}(-1)^{n} \frac{\sin (2 \pi n \phi)}{\sinh \left(n q \pi T / T_{0}\right)}
$$

which is the formula obtained [8]. The periodicity of $I_{\text {Laugh }}$ in $\Phi$ is $\phi_{0}$, which agrees with the general theorem of Byers and Yang [9]. This is due to the presence of the quasiparticle with a fractional charge as argued in Refs. [8]. Since there is no backscattering from impurities in the chiral Tomonaga-Luttinger liquid, the current has no reduction from impurities and therefore shows non-Fermi liquid dependence on the temperature.

For higher hierarchical states, the low temperature dependence turns out to be very different. The dependence of $I(\tau, \phi)$ is plotted in Fig.11 for $\nu=\frac{3}{7}(m=3)$ case and Fig. 2 for $\nu=\frac{4}{9}(m=4)$ case at various temperatures. We see that, as temperature is lowered, the shape of the graph of $I(\tau, \phi)$ continuously changes and exhibits anomalous oscillations. This is very similar to the case of paired FQH states [11]. However the shapes of the graphs become close to the sawtooth function with period $\phi_{0} / 3, \phi_{0} / 4$ respectively. One observes similar behaviors for general $m$. In that case, the period of the sawtooth function at zero temperature is $\phi_{0} / m$ The convergence to the sawtooth function can be analytically proved from the property of $\Theta$. We note that $\Theta\left[\begin{array}{c}0 \\ a / m\end{array}\right](\phi \mid t)=\theta_{3}(\phi+a / m \mid t)$ (Jacobi 3rd theta function) and $\theta_{3}$ is the fundamental solution of the heat equation in time $t$. Thus, as the temperature is lowered, $\Theta\left[\begin{array}{c}0 \\ a / m\end{array}\right](\phi \mid \tau)$ is localized around $\phi=-a / m+\mathbf{Z}$ and the term proportional to $\Theta\left[\begin{array}{c}0 \\ a / m\end{array}\right](\phi \mid t / \nu)$ gives the dominant contribution to $I$ around $\phi=-a / m+\mathbf{Z}$. In the zero temperature limit, it goes to

$$
I \rightarrow-\nu \frac{e v}{k_{B} L}\left(\phi-\frac{a}{m}\right),
$$

for $-\frac{1}{2 m}+\frac{a}{m}<\phi<\frac{1}{2 m}+\frac{a}{m}, \quad a \in \mathbf{Z}$. Note that only at zero temperature the period $\phi_{0} / m$ is realized. Although the period $\phi_{0} / 2$ is common in the BCS superconductivity [9], the period $\phi_{0} / m$ for $m \geq 3$ is rather surprising. To our knowledge, such phenomenon has never been observed in experiments.

Physical explanation of this phenomenon may be given as in Ref. [11]: the condensation in the bulk drives the edge state to be condensated, but the Mermin-Wagner theorem tells that the condensation can occur only at zero temperature. In hierarchical states, the object which supposed to be condensated is the composite boson [18], comprised from the electron and an odd number of flux quantum. The phenomenon we have found provides a new evidence for the exotic condensation in FQH states. The phenomenon clearly shows that the order parameter in hierarchical state has charge $m$. Thus the order parameter should be the $m$-th antisymmetric tensor invariant of $\mathrm{SU}(m)$ composed from $m$ composite bosons. Although the $\mathrm{SU}(m)$ invariant formulation of the order parameter and Landau-Ginzburg theory with $\mathrm{SU}(m)$ Chern-Simons gauge fields are not known, the property of the persistent edge current suggests such formulation exists. On the other hand, as sketched by Moore and Read [14], the extending fields in the bulk rational conformal field theory of FQH state have properties of the order parameter in FQH state. Our results may serve as the evidence of their suggestion from the edge conformal field theory. Chern-Simons theory already has information of condensation.

The persistent edge current should be related to the stability of the FQH state since, in the composite boson picture, the development of plateau arises from the flux piercing which is similar to the vortex state of a type II superconductor. The flux piercing accompanies the persistent edge current as its linear response. For a $m$-th hierarchical state, the flux dependence of the current $I$ at very low temperature is close to the sawtooth function with the period of $1 / m$ to the one for most stable Laughlin states. Accordingly, the maximal amplitude of the current is reduced. It is expected that, at the fluxes where the direction of the current is reversed, the state may be unstabilized, which results in a $1 / m$ narrower plateau. This is consistent with the general claims on hierarchy: as the rank of the hierarchy becomes higher, the state is more unstable [6,5]. Thus the reduction of the amplitude and period of the persistent edge current at zero temperature qualitatively explains the narrower width of plateau for higher hierarchical states.

Experimentally, this phenomenon provides unambiguous means to detect the hierarchy of a given FQH state. In this phenomenon the hierarchy in the FQH state manifests itself in a physical quantity in an unambiguous way.

Finally we would like to comment on states with $\nu=$ $m /(m s-1)$. In that case, it is known that the edge state is not decomposed into chiral and antichiral sectors [16]. Formally the formula for $\nu=m /(m s-1)$ can be obtained by replacing $\chi_{a}^{\mathrm{SU}(m)_{1}}(\tau)$ by $\overline{\chi_{a}^{\mathrm{SU}(m)_{1}}(\tau)}$ in (13). However, as the current has a backscattering and reduction from 
impurities, the formula is not valid at finite temperature.

In conclusion, we investigated the persistent edge current in the hierarchical FQH states. We found that the persistent edge current $I$ is a periodic function of an AB flux with period $\phi_{0}$ and that at zero temperature its period changes to $\phi_{0} / \mathrm{m}$. We also gave a new explanation of the stability of hierarchical states from this property. The experimental observation of the persistent edge current enables one to determine the level $m$ of the hierarchical FQH state in an unambiguous way.

Acknowledgement. We would like to thank M. Kohmoto, D. Lidsky, S. Murakami, J. Shiraishi for various comments and suggestions. We also would like to thank K.Imura for a discussion on the $\nu=\frac{2}{5}$ case at the initial stage of this work.

[1] X.G. Wen, Phys. Rev. B41, 12838(1990); for a review see: X.G. Wen, Int. J. Mod. Phys. B6,1711(1992).

[2] C.L. Kane and M.P.A. Fisher, Phys. Rev. B43, 15233(1992); K. Moon, H. Yi, C. L. Kane, S. M. Girvin and M. P. A.Fisher, Phys. Rev. Lett. 71, 4381 (1993).

[3] F.P.Milliken et al., Solid State Commun. 97, 309 (1996); A.M.Chang et al., Phys. Rev. Lett. 77, 2538
(1996) ;R.de-Picciotto et al., Nature,389, 162 (1997); L.Saminadayar et al., Phys. Rev. Lett. 79, 2526 (1997).

[4] M.Grayson et al. Phys. Rev. Lett. 80,1062(1998).

[5] J.K. Jain, Phys. Rev. Lett. 63, 199 (1989).

[6] F.D.M.Haldane, Phys. Rev. Lett. 51, 645(1983) ; B.I.Halperin, Phys. Rev. Lett. 52,1583(1984) .

[7] C.de.Chamon,D.S.Freed, S.A.Kivelson, S.L.Sondhi and X.G.Wen, Phys. Rev. B55,2331 (1997).

[8] M.R.Geller, D.Loss and G.Kirczenow, Phys. Rev. Lett. 77, 5110(1996); M.R.Geller and D.Loss, Phys. Rev. B56,9692(1997).

[9] N.Byers and C.N.Young, Phys. Rev. Lett. 7,46(1961).

[10] D.J.Thouless and Y.Gefen, Phys. Rev. Lett. 66, 806(1991).

[11] K.Ino, Phys. Rev. Lett. 81,1078(1998).

[12] B.I.Halperin, Helv.Phys.Acta. 56,75(1983).

[13] F.D.M. Haldane and E.H. Rezayi, Phys. Rev. Lett. 60, 956 (1988).

[14] G.Moore and N.Read, Nucl. Phys. B360, 362 (1991).

[15] J. Fröhlich and A. Zee, Nucl. Phys. B364 ,517 (1991) ; X.G. Wen and A. Zee, Phys. Rev. B46 , 2290 (1993).

[16] A. Cappelli, G.V. Dunne, C.A. Trugenberger and G.R. Zemba, Nucl. Phys. B398, 531(1993); A. Cappelli and G.R. Zemba, Nucl. Phys. B490, 595 (1997).

[17] N.D. Mermin and H. Wagner, Phys. Rev. Lett. 17, 1133 (1966).

[18] S.Girvin and A.MacDonald, Phys. Rev. Lett. 58,1252 (1987).

[19] S.C.Zhang, H.Hansson and S.Kivelson, Phys. Rev. Lett. 62,82 (1989); N.Read, Phys. Rev. Lett. 62,86(1989).

[20] N.Read, Phys. Rev. Lett. 65, 1502(1990)

[21] D.Mumford, Tata Lectures on Theta I, (Progress in mathematics vol.28) Birkhäuser. 


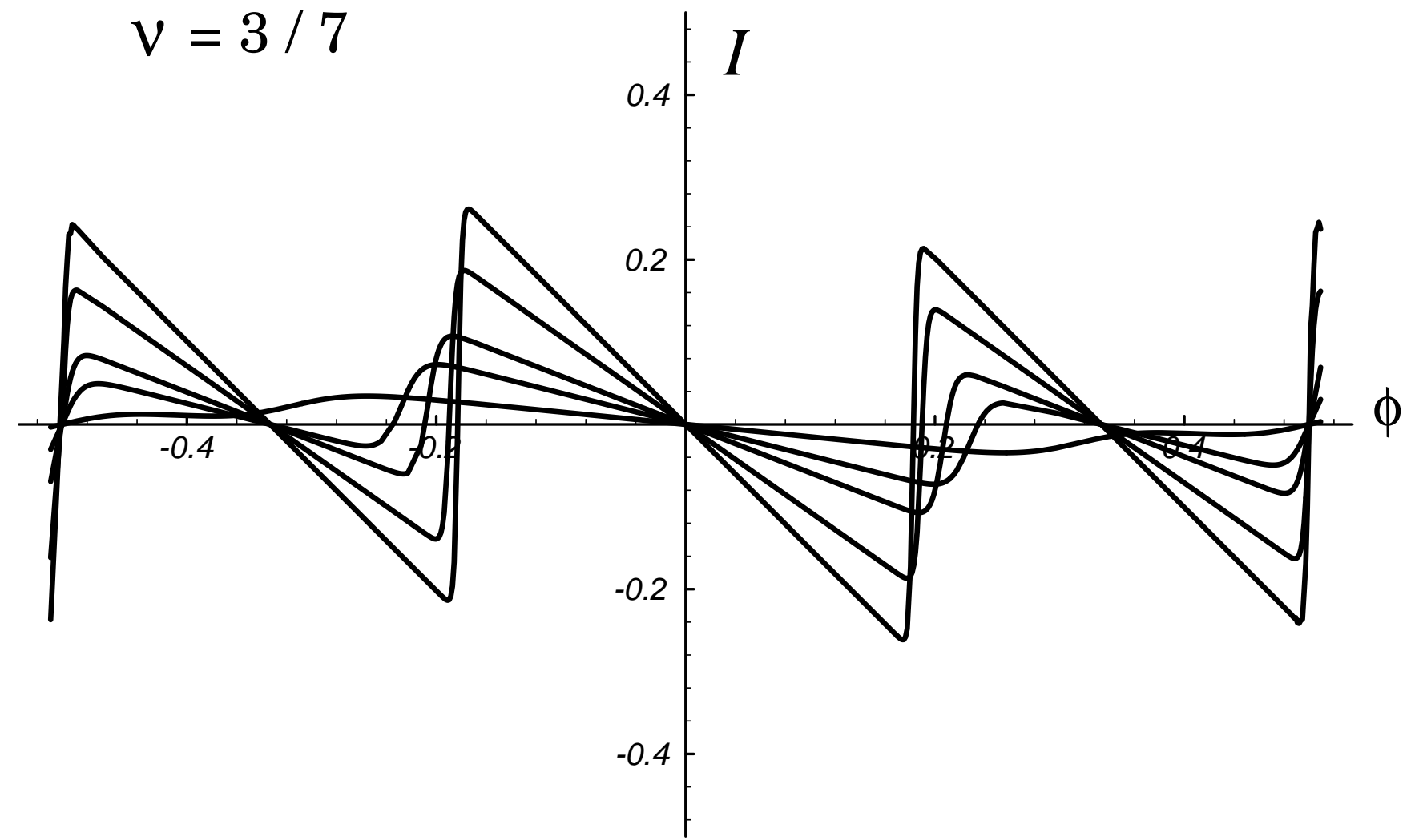

FIG. 1. The flux dependence of the persistent currents for the $\nu=3 / 7$ state $(m=3)$ at temperatures $T / T_{0}=0.29,0.23,0.21,0.18,0.17$. The currents are measured in the unit $\frac{\nu e v}{2 k_{B} L}$. 


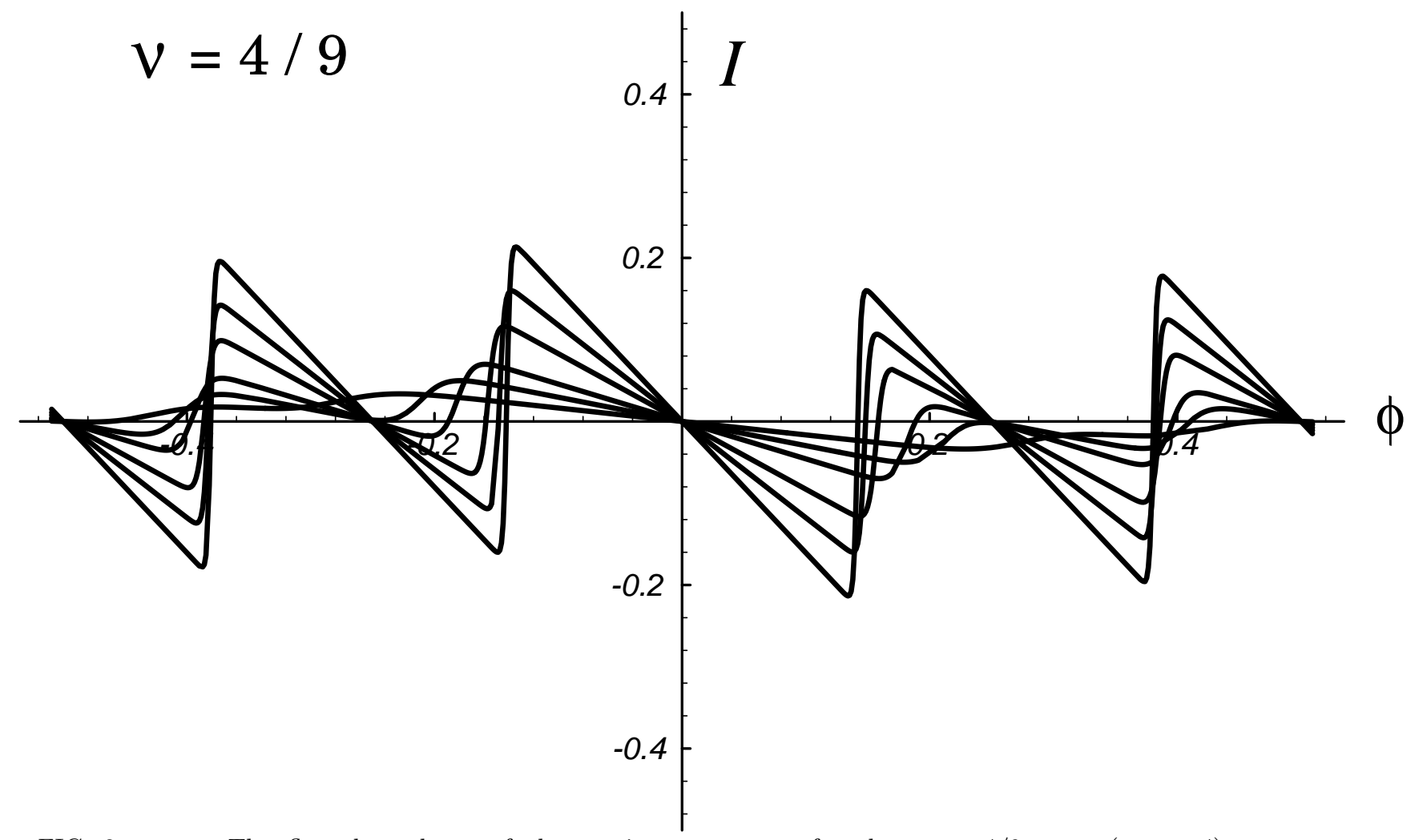

FIG. 2. The flux dependence of the persistent currents for the $\nu=4 / 9$ state $(m=4)$ at temperatures $T / T_{0}=0.29,0.24,0.22,0.20,0.19,0.18$. As the temperature is lowered, the function approaches to a sawtooth function. 